\title{
Nomogram Based on Preoperative Clinical Characteristics to Predict the Risk of Lymph Node Metastasis in Hepatocellular Carcinoma
}

\section{Rongyu Wei}

Laboratory of Hepatobiliary and Pancreatic Surgery, Affiliated Hospital of Guilin Medical University Shuqun Li

Laboratory of Hepatobiliary and Pancreatic Suegery, Affiliated Hospital of Guilin Medical University Liying Ren

Laboratory of Hepatobiliary and Pancreatic Surgery, Affiliated Hospital of Guilin Medical University Junxiong Yu

Laboratory of Hepatobiliary and Surgery, Affiliated Hospital of Guilin Medical University

Weijia Liao ( $\sim$ liaoweijia288@163.com )

Laboratory of Hepatobiliary and Pancreatic Surgery, Affiliated Hospital of Guilin Medical University, Guilin, Guangxi 541001, P.R. China https://orcid.org/0000-0002-8906-8612

\section{Research}

Keywords: HCC, LNM, Prediction, Nomogram

Posted Date: November 20th, 2020

DOI: https://doi.org/10.21203/rs.3.rs-111311/v1

License: (c) (i) This work is licensed under a Creative Commons Attribution 4.0 International License. Read Full License 


\section{Abstract}

Background: There are limitations in judging the occurrence of lymph node metastasis (LNM) in hepatocellular carcinoma (HCC) before surgery. The purpose of this study was to establish a preoperative nomogram for predicting the risk of LNM in HCC and to explore its clinical utility.

Methods: A total of $195 \mathrm{HCC}$ patients undergoing radical hepatectomy were retrospectively analyzed. According to the presence or absence of LNM, the patients were divided into two groups, and the clinical characteristics of the two groups were compared. Risk factors for LNM were assessed based on logistic regression, and a nomogram was established. The receiver operating characteristic (ROC) curve was used to calculate area under the curve (AUC) of the logistic regression model, and the predictive accuracy of the nomogram was evaluated by the concordance index (C-index). The clinical efficacy of the nomogram was detected by decision curve analysis (DCA).

Results: Logistic analysis revealed hepatitis B surface antigen (HBsAg) $(\mathrm{HR}=3.50,95 \% \mathrm{Cl}, 1.30-9.42, \mathrm{P}=$ 0.013), globulin ( $\mathrm{HR}=2.46,95 \% \mathrm{Cl}, 1.05-5.75, \mathrm{P}=0.039)$, neutrophil to lymphocyte ratio $(\mathrm{NLR})(\mathrm{HR}=7.64$, $95 \% \mathrm{Cl}, 3.22-18.11, \mathrm{P}<0.001)$ and tumor size $(\mathrm{HR}=3.86,95 \% \mathrm{Cl}, 1.26-11.88 \mathrm{P}=0.018)$ were independent risk factors for lymph node metastasis in HCC. The nomogram was established based on the above 4 variables, and the AUC was $0.835(95 \% \mathrm{Cl}, 0.780-0.890)$. The calibration curve showed that the model has good predictive ability, and DCA indicates good predictive effect.

Conclusions: The nomogram established by analyzing the preoperative clinical characteristics is a simple tool that can predict the risk of lymph node metastasis in HCC patients and guide clinicians to make better clinical decisions.

\section{Introduction}

Liver cancer is one of the most common malignant tumors in the world, and China has a high incidence of liver cancer, accounting for approximately $50 \%$ of the world's total (1). As the most common type of liver cancer, hepatocellular carcinoma (HCC) is ranked as the sixth most common neoplasm and the second leading cause of cancer death due to its poor prognosis $(2,3)$. In 2018, approximately 841,000 people were diagnosed with liver cancer worldwide, and approximately 781,000 died of liver cancer (3). At present, the main treatment for HCC is surgical removal of the tumor and other adjuvant treatments (such as local radiofrequency ablation, hepatic artery chemoembolization, molecular targeted therapy and radioembolization) (4). Although the treatment of liver cancer has improved, liver cancer still has a low survival rate and high recurrence rate.

Tumor prognosis is affected by multiple factors, and metastasis is generally associated with tumor progression and recurrence and is one of the main causes of death in tumor patients. LNM is an important factor affecting the progression and recurrence of gastrointestinal malignancies (5), and it is the second most common type of extrahepatic metastasis in liver cancer after hematogenous metastasis (6). At present, the specific mechanism of the development of LNM in HCC is not clear, but cumulative 
studies have confirmed that the prognosis of HCC patients with LNM is worse than that without LNM (7). Ultrasound, computed tomography (CT) and magnetic resonance imaging (MRI) can clearly show enlarged lymph nodes in the hilar region before operation, but it is difficult to distinguish whether these are caused by tumor metastasis (8-10) and results in certain limitations in the diagnosis of LNM.

As a graphical display tool based on a regression model, a nomogram shows the probability of target clinical events and provides an individualized highly accurate risk estimation. The aim of this study was to explore the risk factors for LNM in HCC and to analyze and eventually formulate a prediction model for LNM risks.

\section{Patients And Methods}

\section{Patients}

Data from this study were collected from $195 \mathrm{HCC}$ patients who underwent hepatectomy in the Affiliated Hospital of Guilin Medical University from February 2009 to May 2016. They were divided into two groups: a group of patients with the presence of LNM $(n=65)$ and a group of patients with the absence of LNM $(n=130)$ with a patient ratio of 1:2. The inclusion criteria for this study were as follows: $i)$ patients were > 18 years old; ii) all patients with HCC were pathologically confirmed; and iii) open surgery with intraoperative perihepatic lymph node exploration was performed. The exclusion criteria were as follows: i) received other alternative treatments before surgery, such as radiofrequency ablation and injection of anhydrous alcohol; and ii) had secondary liver cancer or HCC combined with other tumors, including gastric cancer and bladder cancer. The evaluated variables were patient demographics, blood tests, tumor characteristics (diameter and number) and pathological examinations. This study was approved by the research ethics committee of the Affiliated Hospital of Guilin Medical University and conformed to the ethical guidelines of the Declaration of Helsinki. Written informed consent was received from all subjects.

The location and surgical methods of lymph node exploration

Perihepatic lymph nodes were routinely probed intraoperatively in all HCC patients, and the scope of exploration included lymph nodes in the hepatoduodenal ligament, retropancreatic space, para-common hepatic artery and celiac trunk (11). If the preoperative or intraoperative exploration results show that the lymph nodes were positive or suspiciously positive, the corresponding lymph nodes were removed by electrocoagulation and sent for pathological examination.

Statistical analysis

All statistical analyses were performed using SPSS (version 26, Chicago, IL, USA) and R (version 3.6.1, R Foundation for Statistical Computing, Vienna, Austria). URL https://www.R-project.org/). Categorical data are expressed as numbers and percentages. Continuous variables were expressed as means \pm standard deviations (SDs). The comparison of categorical variables was evaluated using Student's t-test or the 
Mann-Whitney $\mathrm{U}$ test. Univariate and multivariate logistic regression analyses were used to analyze the risk factors for LNM, calculate the hazard ratios (HRs) of inferred parameters and $95 \%$ confidence intervals (Cls), and establish a nomogram. The predictive accuracy of the models was measured using the concordance index (C-index), by quantifying the level of agreement between the predicted probabilities and the actual possibility of having the event of interest. In the multivariable logistic model, calibration curves were used to evaluate the calibration capability of the model, and the receiver operating characteristic (ROC) curve was used to calculate the area under the curve (AUC) of the logistic regression model. The nomogram was constructed and the calibration curve was drawn using the "rms" package; the "pROC" package was used for ROC curve analyses, and the "rmda" package was used for DCA curve analyses. $P$ value $<0.05$ was considered statistically significant.

\section{Results}

\section{Patient characteristics}

After screening with the inclusion and exclusion criteria, a total of 195 patients with HCC were enrolled, and their data were analyzed. We divided the patients into two groups for comparisons: the presence of LNM cohort and the absence of LNM cohort. Among them, 65 patients were diagnosed with extrahepatic LNM, and 130 patients were diagnosed without LNM. The characteristic parameters are shown in Table 1. There were statistically significant differences in smoking, HBsAg, white blood cell (WBC) level, neutrophil (NEUT) level, lymphocyte count (LYMPH), platelet level, albumin level, globulin level, aspartate aminotransferase (AST) level, alkaline phosphatase (ALP) level, $\gamma$-glutamyl transpeptidase ( $\gamma$-GT) level, NLR level, tumor size and tumor number between the two cohorts $(P<0.05)$. The parameter variable, except the lymphocyte count and albumin level, were higher in the presence of LNM group than in the absence of LNM group; the lymphocyte count and albumin level were slightly lower in the presence of LNM group than in the absence of LNM group.

Independent significant factors for the presence of LNM in HCC

Univariate logistic regression analysis revealed that smoking, HBsAg, albumin level, globulin level, AST level, ALP level, Y-GT level, NLR level, tumor size and tumor number were risk factors for LNM. Ten variables related to LNM in the above univariate analysis were included in multivariate logistic regression analysis. $\mathrm{HBsAg}(\mathrm{HR}=3.50,95 \% \mathrm{Cl}, 1.30-9.42, \mathrm{P}=0.013)$, globulin level $(\mathrm{HR}=2.46,95 \% \mathrm{Cl}, 1.05-5.75, \mathrm{P}=$ $0.039), \mathrm{NLR}(\mathrm{HR}=7.64,95 \% \mathrm{Cl}, 3.22-18.11, \mathrm{P}<0.001)$ and tumor size $(\mathrm{HR}=3.86,95 \% \mathrm{Cl}, 1.26-11.88, \mathrm{P}=$ 0.018) were independently associated with LNM in HCC (Table 2).

Development and validation of a nomogram for preoperative prediction of LNM in HCC

Based on the results of multivariate logistic regression, we included 4 variables, namely, HBsAg, globulin level, NLR level, and tumor size to establish a prediction model to predict the probability of LNM in HCC, which is represented by a nomogram (Figure 1). The AUC value of the nomogram was $0.835(95 \% \mathrm{Cl}$, $0.780-0.890$ ), which was equal to the C-index. When the optimal cutoff value was obtained, the sensitivity 
and specificity were 0.923 and 0.638 , respectively, with an optimal distinction between the two groups (Figure 2). The difference between the predicted value and the corresponding bias correction value was obtained by 1000 samples with bootstrapping; calibration curves (Figure 3) were drawn, which indicated that there was good consistency between the prediction and the actual recurrence of LNM in HCC based on the nomogram.

Clinical utility of the nomogram

Decision curve analysis (DCA) of the nomogram for predicting the presence of LNM is shown in Figure 4. DCA revealed that when the threshold probability was between 0.04 and 0.68 , the net benefit of the nomogram was higher than the benefit of the two extreme curves, indicating that the model can effectively predict the presence of LNM and provide patients with more reasonable clinical treatment decisions.

\section{Discussion}

It is generally believed that $\mathrm{HCC}$ is a common malignant tumor, most of which progressively develops from cirrhosis, atypical hyperplasia (precancerous lesions) and chronic inflammation (12). LNM is a major factor affecting the long-term survival of malignant tumors and plays an important role in the progression and recurrence of HCC. Increasing evidence has shown that the prognosis of patients with LNM is worse than that of patients without LNM (7). Even though studies have indicated that lymph node dissection and radiotherapy have a positive effect on the prognosis of HCC with LNM, the expected OS is only 8.0-14.0 months and 7.0-13.0 months, respectively (13). Compared with lung cancer, esophageal cancer, gastric cancer, bladder cancer and other malignant tumors, HCC has a lower probability of distant metastasis (14). According to domestic and foreign research reports, the intraoperative detection rate of liver cancer $L N M$ is only $0.75 \% \sim 7.5 \%$, while the detection rate in autopsy is as high as $30.3 \%$ (6), which shows that LNM is often ignored by clinicians in clinical work. However, the judgment of LNM in HCC through imaging is not effective, and it is necessary to explore other more sensitive auxiliary indicators to predict LNM risk in HCC patients.

Some previous studies have analyzed the risk factors for HCC complicated with LNM. Zhuang et al. found that CK19 expression and AJCC/UICC T classification were two independent risk factors for LNM in HCC (15). Xiang et al. identified 83 cancer genes that were differentially expressed in lymph nodepositive and lymph node-negative HCC and found that intratumoral hypoxia-inducible factor (HIF) - $1 \mathrm{a}$, vascular endothelial growth factor (VEGF) and matrix metalloproteinase (MMP) -2 were independent risk factors for LNM and can be used to identify high-risk patients with LNM in liver cancer (16). Abnormal expression of IncRNAs has been found to be involved in a variety of biological processes of cancer, including LNM (17-19). For example, Ma et al. suggested that a IncRNA-based nomogram can be used to predict LNM risk (20). Although related studies have revealed some risk factors for LNM in HCC, these indicators need additional testing, postoperative sample testing, and even clinical testing. 
It is well known that HBV infection is one of the main causes of liver cancer. In Eastern countries, a large proportion of the HCC population has a liver background of HBsAg positivity (21). As one of the risk factors for chronic inflammation of the liver, HBV can activate the common pathway for the progression of all liver malignancies (22), which is related to the poor prognosis of HCC (23). Moreover, in intrahepatic cholangiocarcinoma, the incidence of LNM in patients with HBV infection is lower than that in patients without HBV infection, which has been confirmed in some studies (24). Recent studies have reported that HBsAg positivity is also associated with the incidence and progression of other malignancies, such as gastric, esophageal, and pancreatic cancers (25). The increase in serum globulin is associated with a variety of chronic inflammatory diseases, and the increase in inflammatory cytokines in serum globulin contributes to the increase in serum albumin and the inhibition of serum albumin biosynthesis (26). Considering the biological role of inflammation in tumors, some researchers have evaluated the prognosis of tumors by globulin levels and have demonstrated a correlation between globulin and LNM in malignant tumors (27). The lymphocyte-mediated immune response can eliminate abnormal cells and inhibit the production of cytokines for the proliferation and metastasis of various tumors (28). The increase in neutrophils in tumors can lead to an increase in angiogenic factors such as interleukin-8 (29) and vascular endothelial growth factor (VEGF) $(30,31)$ and promote tumor progression. Studies have confirmed that the preoperative NLR has diagnostic value for LNM of bladder cancer (32). Moreover, patients with a higher NLR are more likely to develop LNM in malignant tumors, such as gastric cancer (33), colorectal cancer (34), esophageal cancer (35), and small cell lung cancer (36). Cumulative studies have shown that among HCC patients, the larger the tumor diameter is, the higher the probability of LNM (37), which may be related to the biological invasive ability of the tumor; this conclusion has also been confirmed in studies of malignant tumors such as intrahepatic cholangiocarcinoma (38) and gastric cancer (39).

Nomograms are a common tool for evaluating tumors with high accuracy and applicability (40). In this study, we established a nomogram to predict the risk of preoperative LNM in HCC by including 4 clinical characteristic variables related to tumor LNM (HBsAg level, globulin level, NLR level, and tumor size). This nomogram demonstrated good consistency between predictions and observations and has great predictive value for LNM risk in HCC. Furthermore, DCA is a novel method for evaluating diagnostic tests, predictive models and molecular markers (41). In our research, we evaluated a nomogram through DCA. Fortunately, the model showed good clinical utility within an appropriate range. Although domestic and foreign guidelines for HCC management have not disclosed feasible and effective treatments for LNM in $\mathrm{HCC}$, surgical dissection or radiotherapy of LNM was effective to improve patient survival and prognosis $(8,10)$. In this study, we have provided a simple and accurate predictive tool for preoperative LNM risk assessment of HCC patients, which not only preliminarily determines the clinical stage of the tumor before the operation but also affects the clinical treatment plan and surgical decisions to provide better consultation for patients.

Of course, there are some limitations in this study. First, due to the low probability of LNM occurring in HCC, it is necessary for us to further increase the sample size to verify our study in future studies. Second, this was a retrospective analysis, and inevitably, there were some biases in the selection of patients. 
Third, most of our research subjects were HBV-positive patients, and whether this research is applicable to groups in other regions and countries is not yet known. In addition, our data came from a single center, which requires some prospective studies to further confirm the reliability of the model. We are also currently working to collect data from multiple centers to build a database for external validation of this model. In the future, we may combine this model with other clinical characteristics, such as imaging information, to build a better LNM risk prediction model.

\section{List Of Abbreviations}

HCC, hepatocellular carcinoma; LNM, lymph node metastasis; ROC, receiver operating characteristic; AUC, area under the curve; C-index, concordance index; DCA, decision curve analysis; $\mathrm{HBsAg}$, hepatitis $B$ surface antigen; WBC, white blood cell; NEUT, neutrophils; LYMPH, lymphocyte count; TBIL, total bilirubin; DBIL, direct bilirubin; ALT, alanine aminotransferase; AST, aspartate aminotransferase; ALP, alkaline phosphatase; $Y$-GT, $Y$-glutamyl transpeptidase; AFP, alpha-fetoprotein; NLR, neutrophil to lymphocyte ratio; $\mathrm{HR}$, hazard ratio; $\mathrm{Cl}$, confidence interval; VS, versus.

\section{Declarations}

\section{Ethics approval and consent to participate}

This study was approved by the research ethics committee of the Affiliated Hospital of Guilin Medical University and complied with the Declaration of Helsinki Principles. Informed consents were obtained from all patients.

\section{Consent for publication}

Written informed consent for publication was obtained from all participants.

\section{Availability of data and materials}

All data generated or analysed during this study are included in this published article.

Competing interests

The authors declare that they have no competing interests.

\section{Funding}

This work was supported in part by the National Natural Science Foundation of China (No. 81372163), the National Key Sci-Tech Special Project of China (No.2018ZX10302207) and the Technology Planning Project of Guilin (No. 20190218-1).

Author Contributions 
WL and RW designed the research; SL and WL collected data; RW and LR performed the data analysis and model development; SL composed the first draft of the manuscript. WL commented on and critically revised the manuscript. JY and WL critically edited and reviewed the final draft of the manuscript. All the authors contributed to the conception of the study and approved the final manuscript.

Acknowledgments

Not applicable.

\section{References}

1. Chen W, Zheng R, Baade PD, Zhang S, Zeng H, Bray F, et al. Cancer statistics in China, 2015. CA Cancer J Clin. 2016;66:115-32.

2. Bertuccio P, Turati F, Carioli G, Rodriguez T, La Vecchia C, Malvezzi M, et al. Global trends and predictions in hepatocellular carcinoma mortality. J Hepatol. 2017;67(2):302-9.

3. Siegel RL, Miller KD, Jemal A. Cancer statistics, 2019. CA Cancer J Clin. 2019;69(1): 7-34.

4. Forner A, Reig M, Bruix J. Hepatocellular carcinoma. Lancet. 2018;391: 1301-1314.

5. Huang W, Chen Z, Shang X, Tian D, Wang D, Wu K, et al. Sox12, a direct target of FoxQ1, promotes hepatocellular carcinoma metastasis through up-regulating Twist1 and FGFBP1. Hepatology. 2015;61:1920-33.

6. Pan T, Xie QK, Lv N,Li XS, Mu LW, Wu PH, et al. Percutaneous CT-guided Radiofrequency Ablation for Lymph Node Oligometastases from Hepatocellular Carcinoma: A Propensity Score-matching Analysis. Radiology. 2017;282:259-270.

7. Zeng ZC, Tang ZY, Fan J, Qin LX, Ye SL, Zhou J, et al. Consideration of role of radiotherapy for lymph node metastases in patients with HCC: retrospective analysis for prognostic factors from 125 patients. Int J Radiat Oncol Biol Phys. 2005;63:1067-76.

8. Katyal S, Oliver JH, Peterson MS, Ferris JV, Carr BS, Baron RL. Extrahepatic metastases of hepatocellular carcinoma. Radiology. 2000;216:698-703.

9. Grobmyer SR, Wang L, Gonen M, Fong Y, Klimstra D, D'Angelica M, et al. Perihepatic lymph node assessment in patients undergoing partial hepatectomy for malignancy. Ann Surg. 2006;244:260-4.

10. Kobayashi S, Takahashi S, Kato Y, Gotohda N, Nakagohri T, Konishi M, et al. Surgical treatment of lymph node metastases from hepatocellular carcinoma. J Hepatobiliary Pancreat Sci. 2011;18:55966.

11. Ercolani G, Grazi GL, Ravaioli M, Grigioni WF, Cescon M, Gardini A, et al. The role of lymphadenectomy for liver tumors: further considerations on the appropriateness of treatment strategy. Ann Surg. 2004;239:202-9.

12. Reebye V, Sætrom P, Mintz PJ, Huang KW, Swiderski P, Peng L, et al. Novel RNA oligonucleotide improves liver function and inhibits liver carcinogenesis in vivo. Hepatology. 2014;59:216-27. 
13. Yamashita H, Nakagawa K, Shiraishi K, Tago M, Igaki H, Nakamura N, et al. Radiotherapy for lymph node metastases in patients with hepatocellular carcinoma: retrospective study. J Gastroenterol Hepatol. 2007;22:523-7.

14. Uchino K, Tateishi R, Shiina S, Kanda M, Masuzaki R, Kondo Y, et al. Hepatocellular carcinoma with extrahepatic metastasis: clinical features and prognostic factors. Cancer. 2011;117:4475-83.

15. Zhuang PY, Zhang JB, Zhu XD, Zhang W, Wu WZ, Tan YS, et al. Two pathologic types of hepatocellular carcinoma with lymph node metastasis with distinct prognosis on the basis of CK19 expression in tumor. Cancer. 2008;112:2740-8.

16. Xiang ZL, Zeng ZC, Fan J, Tang ZY, Zeng HY, Gao DM. Gene expression profiling of fixed tissues identified hypoxia-inducible factor-1a, VEGF, and matrix metalloproteinase-2 as biomarkers of lymph node metastasis in hepatocellular carcinoma. Clin Cancer Res. 2011;17:5463-72.

17. Shang C, Wang W, Liao Y, Chen Y, Liu T, Du Q, et al. LNMICC Promotes Nodal Metastasis of Cervical Cancer by Reprogramming Fatty Acid Metabolism. Cancer Res. 2018;78:877-890.

18. He W, Zhong G, Jiang N, Wang B, Fan X, Chen C, et al. Long noncoding RNA BLACAT2 promotes bladder cancer-associated lymphangiogenesis and lymphatic metastasis. J Clin Invest. 2018;128:861-875.

19. Prensner JR, Zhao S, Erho N, Schipper M, lyer MK, Dhanasekaran SM, et al. RNA biomarkers associated with metastatic progression in prostate cancer: a multi-institutional high-throughput analysis of SChLAP1. Lancet Oncol. 2014;15:1469-1480.

20. Ma J, Zhang L, Bian HR, Lu ZG, Zhu L, Yang P, et al. A Noninvasive Prediction Nomogram for Lymph Node Metastasis of Hepatocellular Carcinoma Based on Serum Long Noncoding RNAs. Biomed Res Int. 2019;2019:1710670.

21. Choo SP, Tan WL, Goh BKP, Tai WM, Zhu AX. Comparison of hepatocellular carcinoma in Eastern versus Western populations. Cancer. 2016;122:3430-3446.

22. Lee CH, Chang CJ, Lin YJ, Yeh CN, Chen MF, Hsieh SY. Viral hepatitis-associated intrahepatic cholangiocarcinoma shares common disease processes with hepatocellular carcinoma. Br $\mathrm{J}$ Cancer. 2009;100:1765-70.

23. Beudeker BJB, Groothuismink ZMA, de Man RA, Witjes CDM, van der Eijk AA, Boonstra A. Hepatitis B core-related antigen levels predict recurrence-free survival in patients with HBV associated early stage hepatocellular carcinoma: results from a Dutch long-term follow-up study. J Viral Hepat. 2020; undefined:undefined.

24. Peng NF, Li LQ, Qin X, Guo Y, Peng T, Xiao KY, et al. Evaluation of risk factors and clinicopathologic features for intrahepatic cholangiocarcinoma in Southern China: a possible role of hepatitis B virus. Ann Surg Oncol. 2011;18:1258-66.

25. Tian T, Song C, Jiang L, Dai J, Lin Y, Xu X, et al. Hepatitis B virus infection and the risk of cancer among the Chinese population. Int J Cancer. 2020;147:3075-3084.

26. Gabay C, Kushner I. Acute-phase proteins and other systemic responses to inflammation. N Engl J Med. 1999;340:448-54. 
27. Chi J, Xie Q, Jia J, Liu X, Sun J, Chen J, et al. Prognostic Value of Albumin/Globulin Ratio in Survival and Lymph Node Metastasis in Patients with Cancer: A Systematic Review and Meta-analysis. J Cancer. 2018;9:2341-2348.

28. Ding PR, An X, Zhang RX, Fang YJ, Li LR, Chen G, et al. Elevated preoperative neutrophil to lymphocyte ratio predicts risk of recurrence following curative resection for stage IIA colon cancer. Int J Colorectal Dis. 2010;25:1427-33.

29. Zurek OW, Pallister KB, Voyich JM. Staphylococcus aureus Inhibits Neutrophil-derived IL-8 to Promote Cell Death. J Infect Dis. 2015;212:934-8.

30. Phan VT, Wu X, Cheng JH, Sheng RX, Chung AS, Zhuang G, et al. Oncogenic RAS pathway activation promotes resistance to anti-VEGF therapy through G-CSF-induced neutrophil recruitment. Proc Natl Acad Sci USA. 2013;110:6079-84.

31. Ohki Y, Heissig B, Sato Y, Akiyama H, Zhu Z, Hicklin DJ, et al. Granulocyte colony-stimulating factor promotes neovascularization by releasing vascular endothelial growth factor from neutrophils. FASEB J. 2005;19:2005-7.

32. Tang X, Cao Y, Liu J, Wang S, Yang Y, Du P. Diagnostic and Predictive Values of Inflammatory Factors in Pathology and Survival of Patients Undergoing Total Cystectomy. Mediators Inflamm. 2020;2020:9234067.

33. Wang K, Diao F, Ye Z,Zhang X, Zhai E, Ren H, et al. Prognostic value of systemic immuneinflammation index in patients with gastric cancer. Chin J Cancer. 2017;36:75.

34. Mazaki J, Katsumata K, Kasahara K, Tago T, Wada T, Kuwabara H, et al. Neutrophil-to-lymphocyte ratio is a prognostic factor for colon cancer: a propensity score analysis. BMC Cancer. 2020;20:922.

35. Zhou SB, Guo XW, Gu L, Ji SJ. Influential factors on radiotherapy efficacy and prognosis in patients with secondary lymph node metastasis after esophagectomy of thoracic esophageal squamous cell carcinoma. Cancer Manag Res. 2018;10:217-225.

36. Wang $H$, Zhao J, Zhang M, Han L, Wang M, Xingde L. The combination of plasma fibrinogen and neutrophil lymphocyte ratio (F-NLR) is a predictive factor in patients with resectable non small cell lung cancer. J Cell Physiol. 2018;233:4216-4224.

37. Yuki K, Hirohashi S, Sakamoto M, Kanai T, Shimosato Y. Growth and spread of hepatocellular carcinoma. A review of 240 consecutive autopsy cases. Cancer. 1990;66:2174-2179.

38. Orimo T, Kamiyama T, Mitsuhashi T, Kamachi H, Yokoo H, Wakayama K, et al. Impact of tumor localization on the outcomes of surgery for an intrahepatic cholangiocarcinoma. J Gastroenterol. 2018;53:1206-1215.

39. Murai K, Takizawa K, Shimoda T, Fujii S, Sugino T, Yoshida M, et al. Effect of double-layer structure in intramucosal gastric signet-ring cell carcinoma on lymph node metastasis: a retrospective, singlecenter study. Gastric Cancer. 2019;22:751-758.

40. Zhang Z, Kattan MW. Drawing Nomograms with R: applications to categorical outcome and survival data. Ann Transl Med. 2017;5:211. 
41. Vickers AJ, Cronin AM, Elkin EB, Gonen M. Extensions to decision curve analysis, a novel method for evaluating diagnostic tests, prediction models and molecular markers. BMC Med Inform Decis Mak. 2008;8:53

\section{Tables}

Table 1. Preoperative clinical characteristics in absent and present of LNM. 


\begin{tabular}{|c|c|c|c|c|}
\hline \multicolumn{2}{|l|}{ Characteristics } & \multicolumn{2}{|c|}{ Lymph node metastasis } & \multirow[t]{2}{*}{$P$ value } \\
\hline & & \multirow{2}{*}{$\begin{array}{l}\text { Absent }(n=130) \\
50.42 \pm 12.03\end{array}$} & Present $(n=65)$ & \\
\hline Age (years) & & & $51.67 \pm 12.44$ & 0.498 \\
\hline \multirow[t]{2}{*}{ Sex } & Men (n) & $110(84.6 \%)$ & $55(84.6 \%)$ & \multirow[t]{2}{*}{1.000} \\
\hline & Women (n) & $20(15.4 \%)$ & $10(15.4 \%)$ & \\
\hline \multirow[t]{2}{*}{ Family history } & Present (n) & $11(8.5 \%)$ & $10(15.4 \%)$ & \multirow[t]{2}{*}{0.142} \\
\hline & Absent (n) & 119 (91.5\%) & $55(82.6 \%)$ & \\
\hline \multirow[t]{2}{*}{ Smoking } & Present (n) & $52(40 \%)$ & $37(56.9 \%)$ & \multirow[t]{2}{*}{0.025} \\
\hline & Absent (n) & $78(60 \%)$ & $28(43.1 \%)$ & \\
\hline \multirow[t]{2}{*}{ Alcohol abuse } & Present (n) & $53(40.8 \%)$ & $34(52.3 \%)$ & \multirow[t]{2}{*}{0.127} \\
\hline & Absent (n) & $77(59.2 \%)$ & $31(47.7 \%)$ & \\
\hline \multirow[t]{2}{*}{ Cirrhosis } & Present (n) & $118(90.8 \%)$ & $60(92.3 \%)$ & \multirow[t]{2}{*}{0.720} \\
\hline & Absent (n) & $12(9.2 \%)$ & $5(7.7 \%)$ & \\
\hline \multirow[t]{2}{*}{ HBsAg } & Positive (n) & $114(87.7 \%)$ & $47(72.3 \%)$ & \multirow[t]{2}{*}{0.008} \\
\hline & Negative (n) & $16(12.3 \%)$ & $18(27.7 \%)$ & \\
\hline \multicolumn{2}{|l|}{ WBC $\left(\times 10^{9} / \mathrm{L}\right)$} & $6.12 \pm 1.93$ & $7.94 \pm 2.97$ & $<0.001$ \\
\hline \multicolumn{2}{|l|}{ NEUT $\left(\times 10^{9} / \mathrm{L}\right)$} & $3.61 \pm 1.57$ & $5.53 \pm 2.70$ & $<0.001$ \\
\hline \multicolumn{2}{|l|}{ LYMPH $\left(\times 10^{9} / \mathrm{L}\right)$} & $1.77 \pm 0.59$ & $1.54 \pm 0.53$ & 0.010 \\
\hline \multicolumn{2}{|c|}{ Platelets $\left(\times 10^{9} / \mathrm{L}\right)$} & $178.17 \pm 71.64$ & $221.19 \pm 100.50$ & 0.001 \\
\hline \multicolumn{2}{|l|}{ Albumin $(\mathrm{g} / \mathrm{L})$} & $39.15 \pm 4.25$ & $37.41 \pm 6.39$ & 0.025 \\
\hline \multicolumn{2}{|l|}{ Globulin (g/L) } & $30.67 \pm 5.31$ & $33.99 \pm 8.21$ & 0.001 \\
\hline \multicolumn{2}{|l|}{ TBIL $(\mu \mathrm{mol} / \mathrm{L})$} & $16.33 \pm 7.95$ & $17.00 \pm 15.51$ & 0.690 \\
\hline \multicolumn{2}{|l|}{$\mathrm{DBIL}(\mu \mathrm{mol} / \mathrm{L})$} & $5.99 \pm 3.00$ & $7.39 \pm 7.27$ & 0.058 \\
\hline \multicolumn{2}{|l|}{ ALT (U/L) } & $45.38 \pm 42.64$ & $47.73 \pm 43.36$ & 0.719 \\
\hline \multicolumn{2}{|l|}{ AST (U/L) } & $49.20 \pm 39.15$ & $67.27 \pm 45.88$ & 0.005 \\
\hline \multicolumn{2}{|l|}{$\operatorname{ALP}(\mathrm{U} / \mathrm{L})$} & $91.70 \pm 34.67$ & $137.75 \pm 66.52$ & $<0.001$ \\
\hline \multicolumn{2}{|c|}{ Y-GT (U/L): median (IQR) } & $73.8(44.25-128.06)$ & $158.48(80.97-251.37)$ & $<0.001$ \\
\hline \multicolumn{2}{|c|}{$\operatorname{AFP}(\mathrm{ng} / \mathrm{ml})$ : median (IQR) } & $356.0(21.57-1210.0)$ & $261.9(5.79-1210.0)$ & 0.786 \\
\hline \multicolumn{2}{|l|}{ NLR level } & $2.21 \pm 1.28$ & $3.81 \pm 2.12$ & $<0.001$ \\
\hline
\end{tabular}




\begin{tabular}{|lllll|}
\hline Tumor size $($ range, cm) & $7.09 \pm 4.34$ & $10.87 \pm 4.31$ & $<0.001$ \\
\hline \multirow{2}{*}{ Tumor number } & Multiple (n) & $26(20 \%)$ & $26(40 \%)$ & 0.003 \\
\cline { 2 - 5 } & Single (n) & $104(80 \%)$ & $39(60 \%)$ & \\
\hline
\end{tabular}

n, number of patients; HBsAg, hepatitis B surface antigen; WBC, white blood cell; NEUT, neutrophils; LYMPH, lymphocyte count; TBIL, total bilirubin; DBIL, direct bilirubin; ALT, alanine aminotransferase; AST, aspartate aminotransferase; ALP, alkaline phosphatase; $Y$-GT, $Y$-glutamyl transpeptidase; AFP, alphafetoprotein; NLR, neutrophil to lymphocyte ratio.

Table 2. Independent signifificant factors for LNM identified by logistic regression analysis. 


\begin{tabular}{|c|c|c|c|c|c|c|}
\hline \multirow[t]{2}{*}{ Variable } & \multicolumn{3}{|c|}{ Univariate analysis } & \multicolumn{3}{|c|}{ Multivariate analysis } \\
\hline & HR & $95 \% \mathrm{Cl}$ & $p$ value & HR & $95 \% \mathrm{Cl}$ & $p$ value \\
\hline Age, yeas $(>55 v s \leq 55)$ & 0.96 & $0.52-1.80$ & 0.916 & & & \\
\hline Sex (men $v s$ women) & 1.00 & $0.44-2.28$ & 1.000 & & & \\
\hline Family history (positive vs negative) & 1.96 & $0.78-4.90$ & 0.147 & & & \\
\hline Smoking (present $v s$ absent) & 1.98 & $1.08-3.62$ & 0.026 & & & \\
\hline Alcohol abuse (present $v s$ absent) & 1.59 & $0.87-2.90$ & 0.128 & & & \\
\hline Cirrhosis (positive vs negative) & 1.22 & $0.41-3.62$ & 0.720 & & & \\
\hline HBsAg (positive vs negative) & 2.72 & $1.28-5.80$ & 0.009 & 3.50 & $1.30-9.42$ & 0.013 \\
\hline Albumin, $\mathrm{g} / \mathrm{L}(\leq 35$ vs $>35)$ & 2.38 & $1.20-4.71$ & 0.013 & & & \\
\hline Globulin, g/L (> 33 vs $\leq 33$ ) & 2.41 & $1.30-4.44$ & 0.005 & 2.46 & $1.05-5.75$ & 0.039 \\
\hline TBIL, $\mu \mathrm{mol} / \mathrm{L}(>21 v s \leq 21)$ & 1.05 & $0.51-2.17$ & 0.901 & & & \\
\hline $\mathrm{DBIL}, \mu \mathrm{mol} / \mathrm{L}(>6 v s \leq 6)$ & 1.22 & $0.62-2.40$ & 0.560 & & & \\
\hline ALT, U/L (> $38 v s \leq 38)$ & 1.07 & $0.58-1.95$ & 0.838 & & & \\
\hline AST, U/L (> $40 v s \leq 40)$ & 2.33 & $1.26-4.31$ & 0.007 & & & \\
\hline ALP, U/L (> $90 v s \leq 90)$ & 3.45 & $1.81-6.57$ & $<0.001$ & & & \\
\hline $\mathrm{v}-\mathrm{GT}, \mathrm{U} / \mathrm{L}(>45 v s \leq 45)$ & 2.93 & $1.22-7.04$ & 0.016 & & & \\
\hline AFP, ng/ml (> $20 v s \leq 20)$ & 0.74 & $0.38-1.42$ & 0.361 & & & \\
\hline $\operatorname{NLR}(>2.31 v s \leq 2.31)$ & 7.11 & $3.55-14.27$ & $<0.001$ & 7.64 & $3.22-18.11$ & $<0.001$ \\
\hline Tumor size, $\mathrm{cm}(>5 v s \leq 5)$ & 7.67 & $3.09-18.01$ & $<0.001$ & 3.86 & $1.26-11.88$ & 0.018 \\
\hline Tumor number (multiple $v s$ single) & 2.66 & $1.38-5.14$ & 0.003 & & & \\
\hline
\end{tabular}

$\mathrm{HR}$, hazard ratio; $\mathrm{Cl}$, confidence interval; HBsAg, hepatitis B surface antigen; TBIL, total bilirubin; DBIL, direct bilirubin; ALT, alanine aminotransferase; AST, aspartate aminotransferase; ALP, alkaline phosphatase; $\gamma$-GT, $\gamma$-glutamyl transpeptidase; AFP, alpha-fetoprotein; NLR, neutrophil to lymphocyte ratio.

\section{Figures}


Points

$\begin{array}{lllllllllll}0 & 10 & 20 & 30 & 40 & 50 & 60 & 70 & 80 & 90 & 100\end{array}$

Tumor Size

$>=5 \mathrm{~cm}$

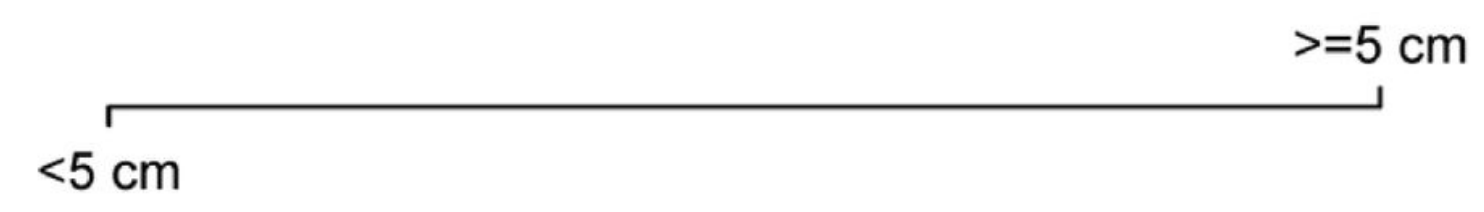

HBsAg

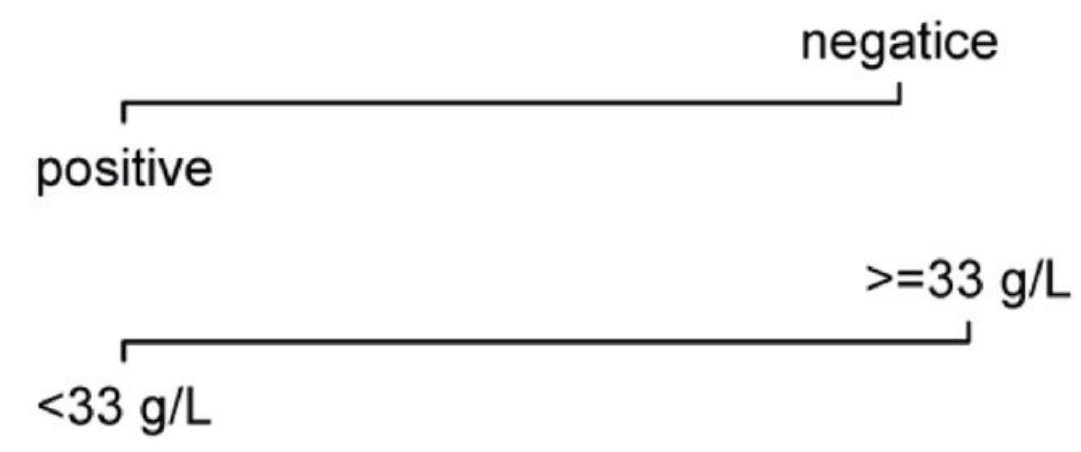

NLR

Globulin

Total Points

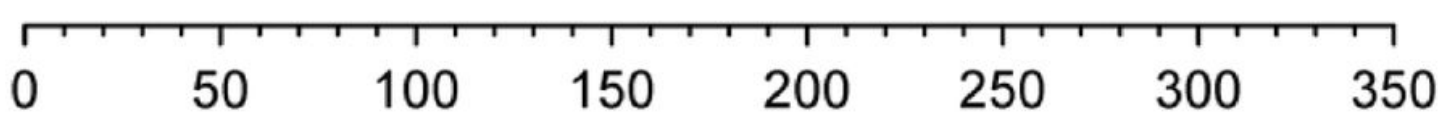

Lymph node metastasis

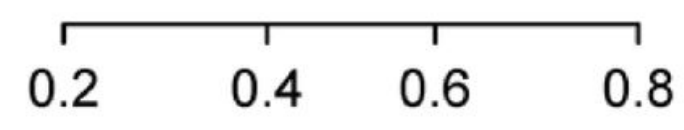

Figure 1

Nomogram for predicting the probability of LNM in HCC on a scale of 0 to 350 . For each covariate, please draw a vertical line upwards to determine the corresponding points. The total points projected on the bottom scales are used to estimate LNM probabilities in individuals. 
Points

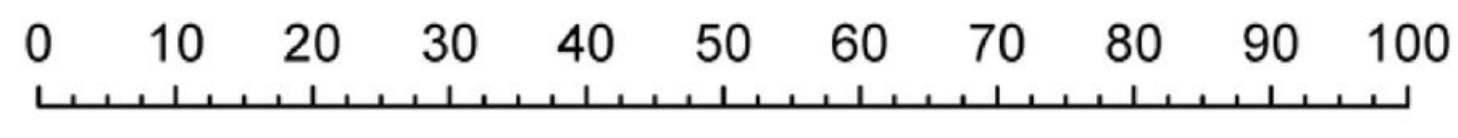

Tumor Size

$>=5 \mathrm{~cm}$

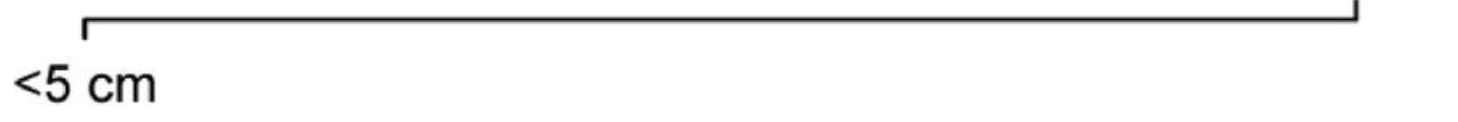

HBsAg

negatice

positive

Globulin

$$
>=33 \mathrm{~g} / \mathrm{L}
$$$$
<33 \mathrm{~g} / \mathrm{L}
$$

NLR

$<2.31$

\section{Total Points}

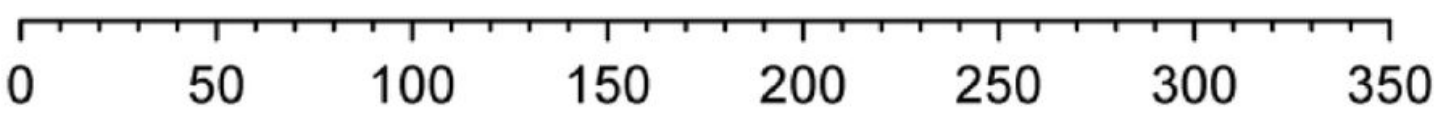

Lymph node metastasis

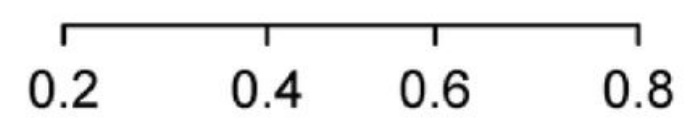

Figure 1

Nomogram for predicting the probability of LNM in HCC on a scale of 0 to 350 . For each covariate, please draw a vertical line upwards to determine the corresponding points. The total points projected on the bottom scales are used to estimate LNM probabilities in individuals. 


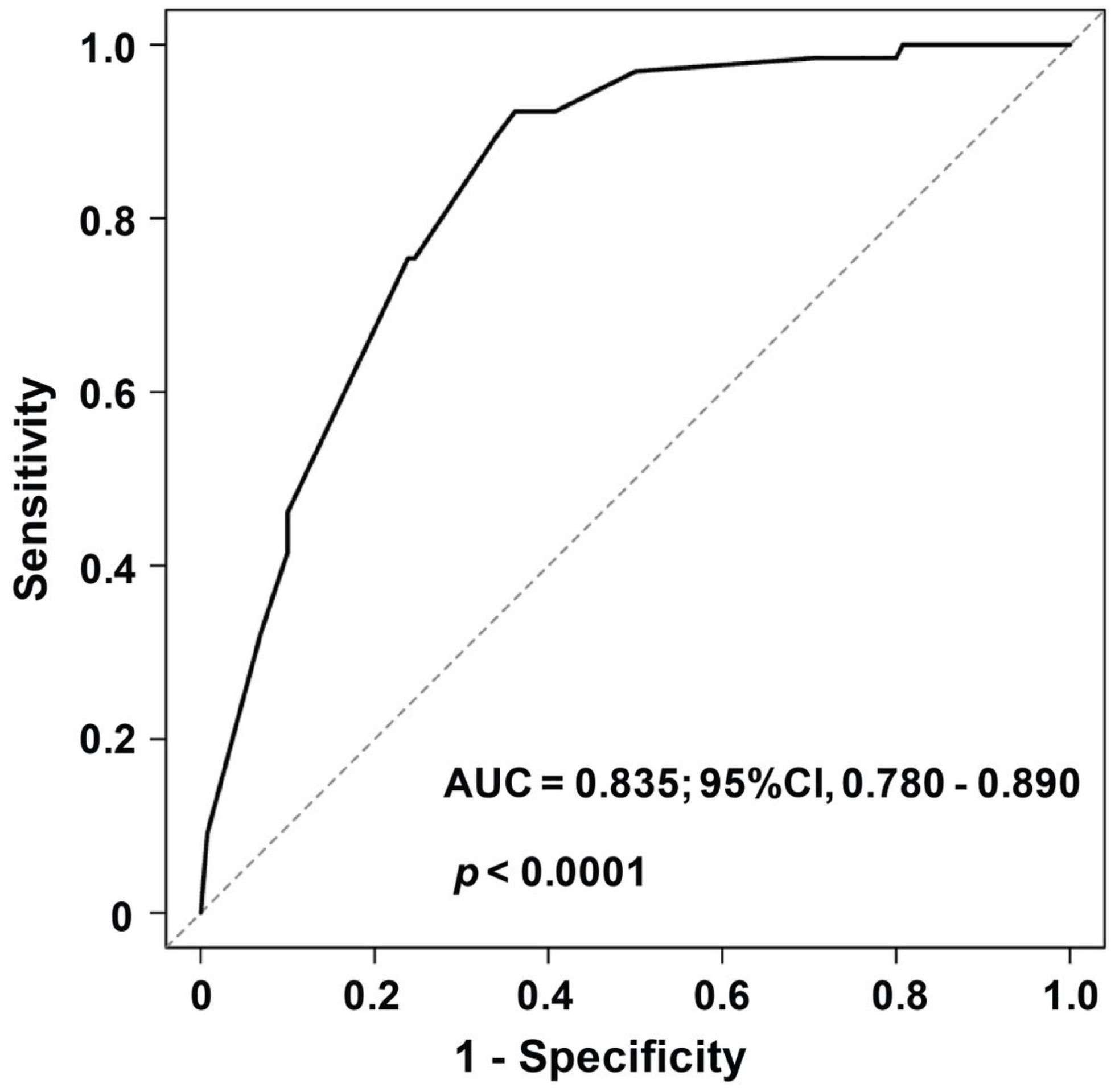

Figure 2

Receiver operating characteristic (ROC) curves for predicting the probability of LNM in HCC patients. 


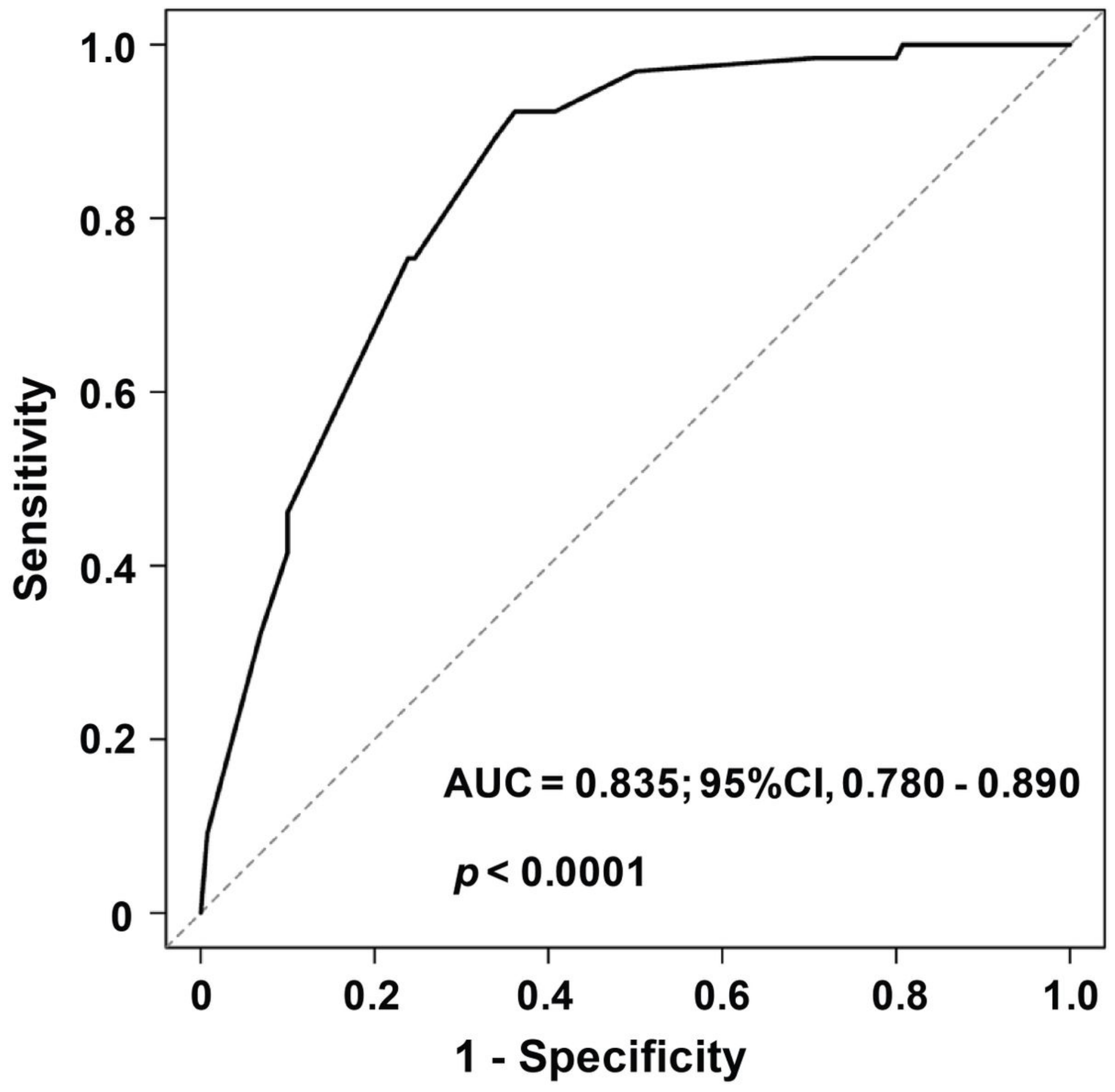

Figure 2

Receiver operating characteristic (ROC) curves for predicting the probability of LNM in HCC patients. 


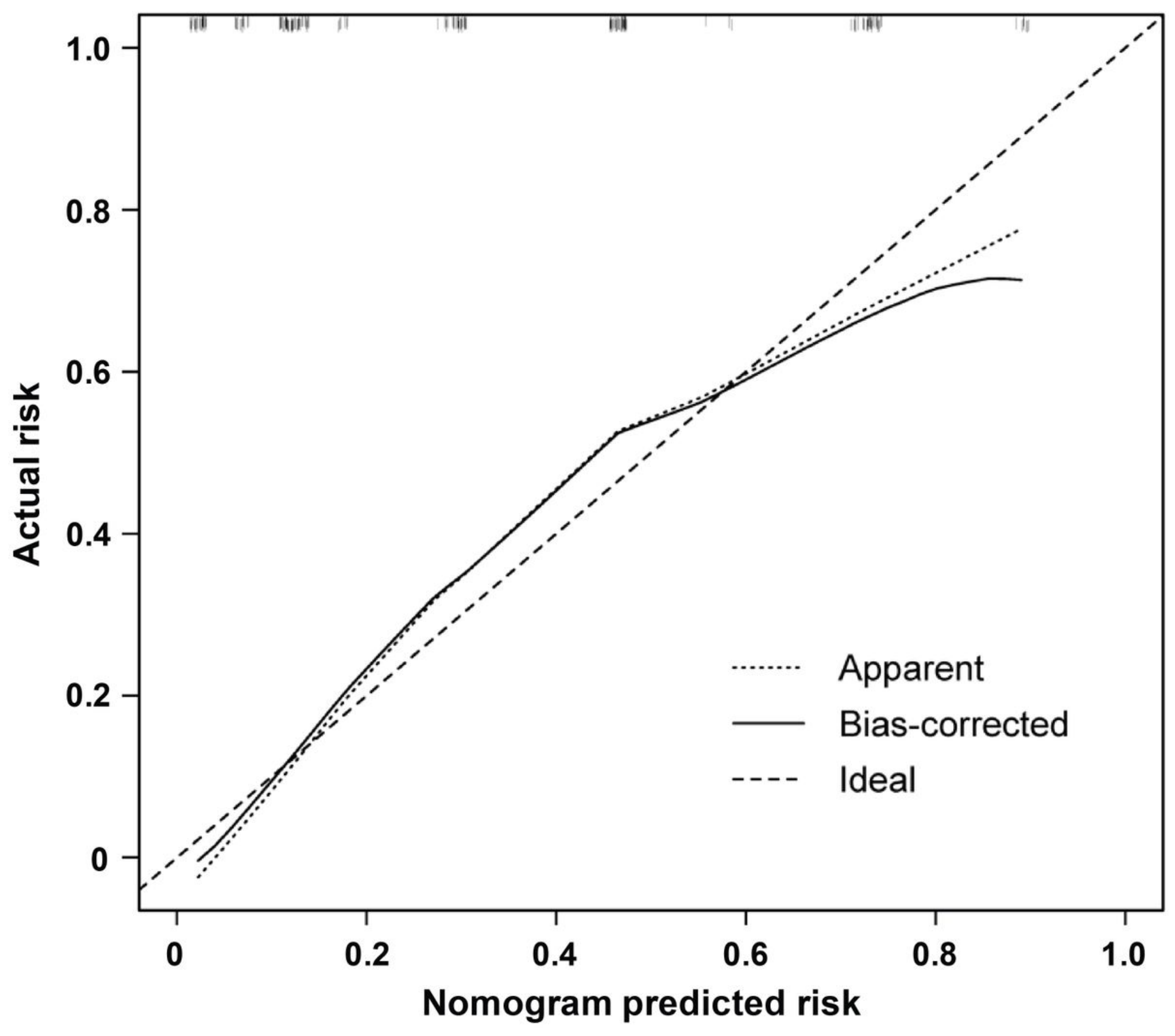

Figure 3

Calibration curve for the nomogram. The x-axis represents the nomogram-predicted probability of LNM in HCC. The y-axis represents the actual probability of LNM in HCC. The ideal line is the estimated probabilities that correspond to the actual observations. The apparent line is the prediction capability of the model obtained after data analysis. The bias-corrected line is the prediction capability of the model obtained after bootstrap correction. Vertical lines at the top of the figure represent the number of patients. The closer the calibration curve is to the diagonal line, the higher the prediction accuracy of the model. 


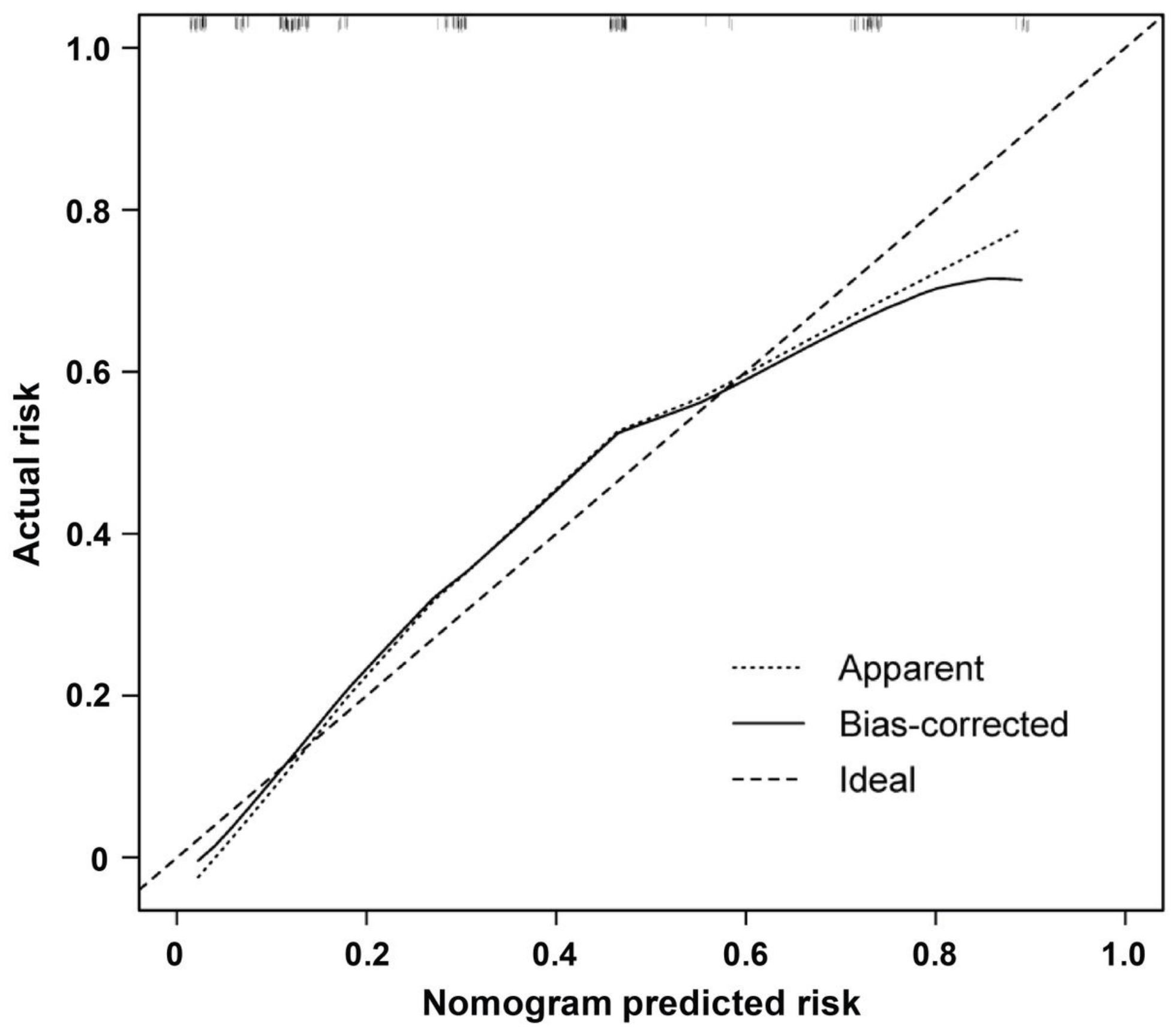

Figure 3

Calibration curve for the nomogram. The x-axis represents the nomogram-predicted probability of LNM in HCC. The y-axis represents the actual probability of LNM in HCC. The ideal line is the estimated probabilities that correspond to the actual observations. The apparent line is the prediction capability of the model obtained after data analysis. The bias-corrected line is the prediction capability of the model obtained after bootstrap correction. Vertical lines at the top of the figure represent the number of patients. The closer the calibration curve is to the diagonal line, the higher the prediction accuracy of the model. 


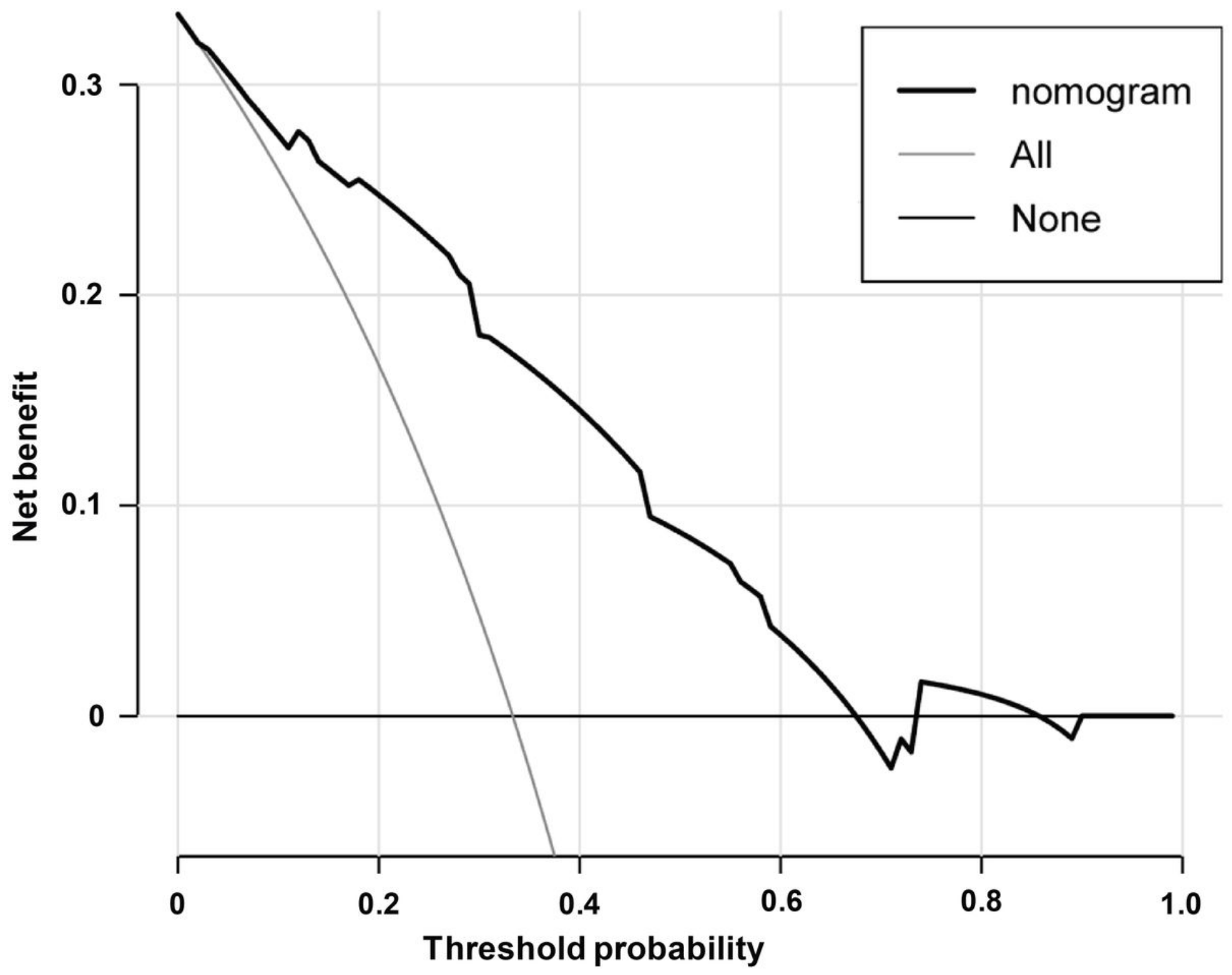

Figure 4

The decision curve of the nomogram for predicting the presence of LNM in HCC. The y-axis represents the net benefit, and the $x$-axis shows the threshold probability. The horizontal solid black line represents the hypothesis that none of the patients experience LNM, and the solid gray line represents the hypothesis that all patients met the endpoint. 


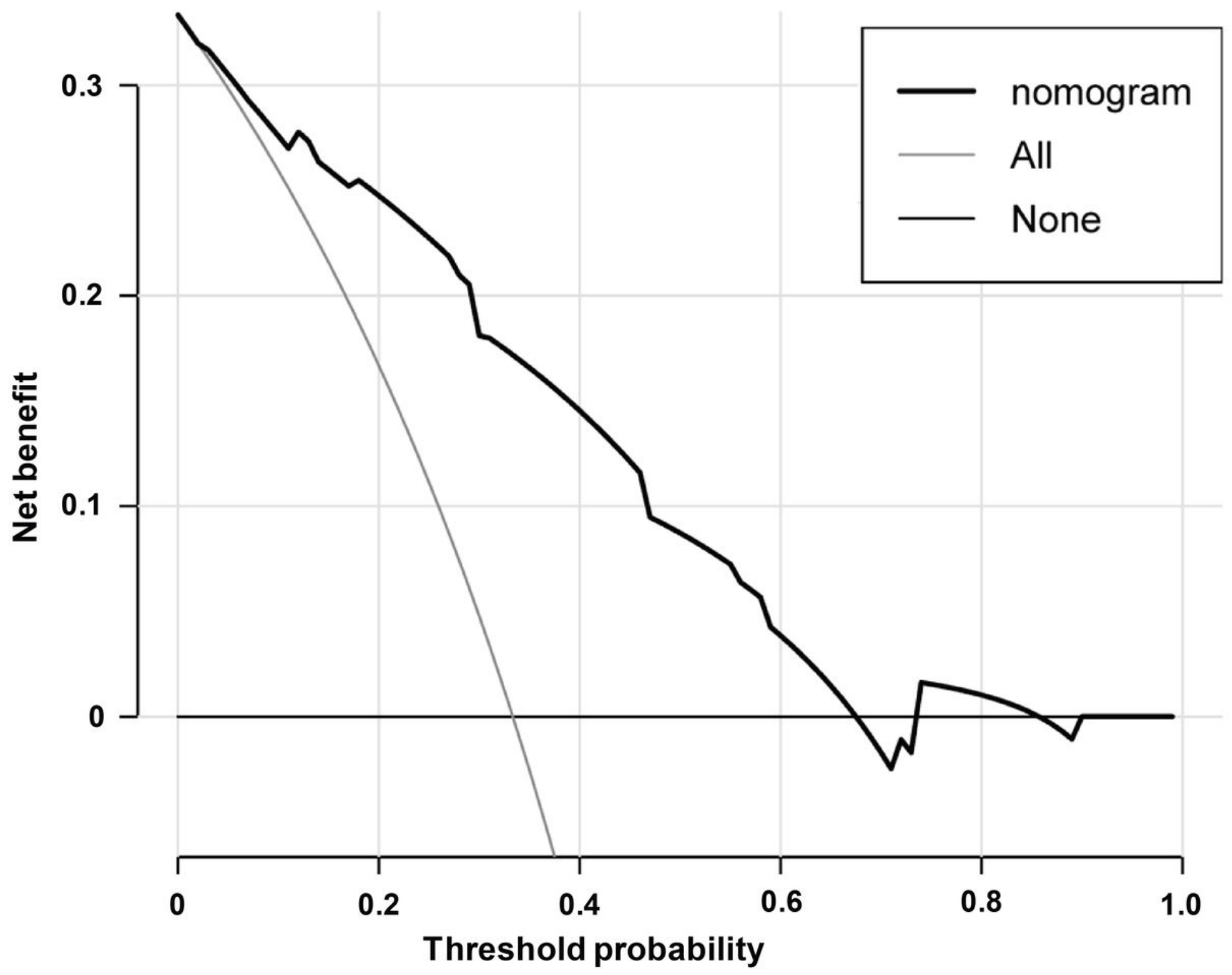

Figure 4

The decision curve of the nomogram for predicting the presence of LNM in HCC. The y-axis represents the net benefit, and the $x$-axis shows the threshold probability. The horizontal solid black line represents the hypothesis that none of the patients experience LNM, and the solid gray line represents the hypothesis that all patients met the endpoint. 